\title{
A CASE OF MULTIPLE STEADY STATES IN REACTION-DIFFUSION SYSTEMS $\left(^{*}\right)$
}

\author{
M. G. VELARDE \\ Departamento de Física, C-3 \\ Universidad Autónoma de Madrid \\ Cantoblanco, Madrid, Spain
}

\begin{abstract}
Résumé. -- Les états stationnaires d'un modèle autocatalytique d'un système réaction-diffusion avec une loi de saturation de Michaelis-Menten (premier ordre Hinshelwood-Langmuir) sont construits dans le cas d'une géométrie unidimensionnelle. Une approximation est utilisée pour convertir le système en un système hamiltonien.

Abstract. - The steady states of a model autocatalytic reaction-diffusion system with MichaelisMenten (first-order Hinshelwood-Langmuir) saturation law are constructed in the case of a onedimensional geometry. An approximation is used to convert the system in a Hamiltonian flow.
\end{abstract}

1. Introduction. - We consider the following model [1]

$$
\begin{gathered}
A \rightarrow Y \\
X+Y \rightarrow 2 X \\
\text { s } \\
X \rightarrow P
\end{gathered}
$$

in which $S$ accounts for the saturation law (MichaelisMenten) given below. $X$ and $Y$ denote intermediate products whereas $A$ and $P$ are externally controllable reactants. The differential problem for model (1) is in dimensionless form

$$
\begin{aligned}
& \frac{\partial}{\partial t} X=X Y-\frac{X}{1+q X}+D_{X} \Delta X \\
& \frac{\partial}{\partial t} Y=A-X Y+D_{Y} \Delta Y
\end{aligned}
$$

in which the quantities $A, X$ and $Y$ account for concentrations, $D_{X}$ and $D_{Y}$ denote diffusion constants, and $q>0$ yields the relative strength of the saturation law in (1) and (2).

In a generic case for either fixed concentrations on boundaries (Dirichlet problem) or fixed fluxes (Neumann problem) the system (2) has a fixed point (a steady homogeneous state) located at

$$
\begin{aligned}
& X_{\mathrm{S}}=A /(1-q A) \\
& Y_{\mathrm{S}}=1-q A .
\end{aligned}
$$

This fixed point becomes unstable for certain values of $A$ and $q[1,2,3]$. We shall describe now the case of multiple steady states in a $d=1$ problem, that bifurcate from (3) on changing the external constraints $A$, $D_{X}, D_{Y}$ and $q$.

(*) Supported by a grant from the Instituto de Estudios Nucleares (Spain).
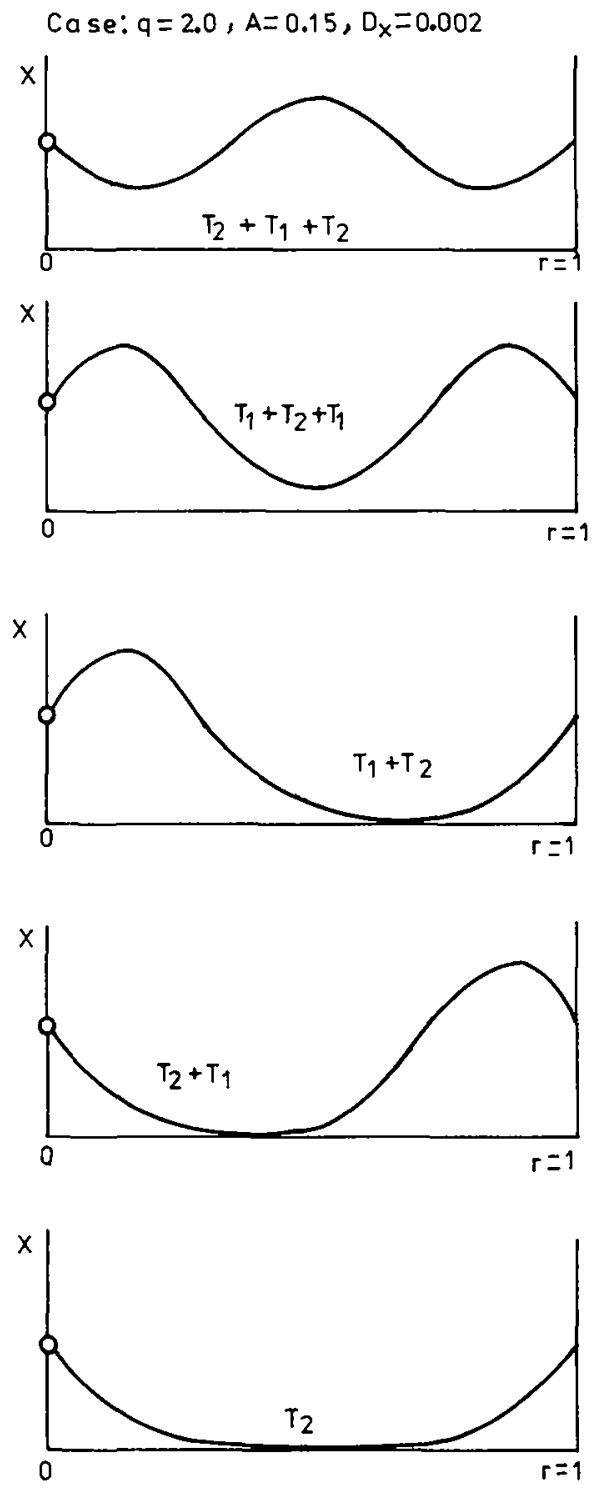

FIG. 1. - The five non-uniform steady states available for $q=2.0$, $D_{x}=0.002$, and $A=0.15$. All but the state $\mathrm{T}_{2}$ (bottom of figure) are unstable. 
2. Multiple steady states. - On a one-dimensional geometry, the limit $D_{Y} \gg D_{X}, D_{Y} \rightarrow \infty, D_{X}<\propto$ has been considered [2]. If we take $r$ as a time variable $(t)$ the differential system originated from (2) defines a Hamiltonian flow. The energy is $\left({ }^{1}\right)$

$$
H(X, V, t)=\frac{V^{2}}{2 D_{\mathrm{X}}}+\frac{X^{2}(1-q A)}{2}-\frac{X}{q}+\lg \frac{|1+q X|}{q^{2}}
$$

in which $V=D \frac{\mathrm{d} X}{\mathrm{~d} t}$. In more compact form

$$
\frac{\mathrm{d}}{\mathrm{d} t}\left(\frac{X}{V}\right)=\left(\begin{array}{r}
\partial H / \partial V \\
-\partial H / \partial X
\end{array}\right)
$$

The Poincaré-Linstedt method and phase-plane techniques have been used to construct the steady states of (4). The system (4) has two fixed points : $X^{(1)}=V^{(1)}=0$, and $X^{(2)}=A(1-q A)^{-1}, V^{(2)}=0$. The existence of a first integral forbids asymptotic

( $\left.{ }^{1}\right)$ Due to the structure of equations (2) in the limit $D_{Y} \rightarrow \infty$, there is always an obvious first integral like (4a). stability of these two fixed points. Nor can they be nodes or focuses. Rather $\left(X^{(1)}, V^{(1)}\right)$ is saddle and $\left(X^{(2)}, V^{(2)}\right)$ is center.

With Dirichlet b.c. (fixed concentrations at boundaries) the only trajectories that are solutions of $(4 b)$ are those for which, if at the origin we have

$$
X(r=0)=X^{(2)} \text { and } V=V(0)
$$

then at the end-point the value is $X(r=1)=X^{(2)}$ with $V=V(r=1)$. These are the trajectories that after any number of turns in a unit-distance end at $X^{(2)}$ though $V$ may be different from $V(0)$. As an illustration of the method employed, we have constructed the five steady nonuniform concentration profiles that correspond to $q=2.0, D_{X}=0.002$ and $A=0.15$ (see figure 1).

Thus we have obtained the classification of all admissible spatially nonuniform structures shown by the system (2) in the limit $D_{Y} \gg D_{X}$. Of these, a computer run permits one to distinguish those that are unstable [2]. Some related work can be found in refs $[3,4]$ and a general introduction to the subject is the recent monograph by Nicolis and Prigogine [5].

\section{References}

[1] Ibañez, J. L., Fatren, V. and Velarde, M. G., Phys. Lett. 58A (1976) 364

[2] Ibã̃̃z, J. L. and Velarde, M. G., J. Math. Phys. 19 (1978) 151. For the stability analysis see : IBANEz, J. L. and Velarde, M. G., Phys. Rev. A (July 1978).

[3] IBā̃ez, J. L. and Velarde, M. G., J. Non-Equilib. Therm. 3 (1978) 63.
[4] Ibañez, J. L., Fairen, V. and Velarde, M. G., Phys. Lett. 59 (1976) 335; Hemmer, P. C. and Velarde, M. G., $Z$. Phys. $B$ (to appear); Fairen, V. and Velarde, M. G., Rep. Math. Phys. (submitted)

[5] Nicours, G. and Prigogine, I., Self-organization in Nonequilibrium systems (Wiley, N.Y.) (1977). 